Helgoländer wiss. Meeresunters. 21, 9-20 (1970)

\title{
Über Massenvorkommen verschiedener makrobenthaler Wirbelloser während der Wiederbesiedlungsphase nach Schädigungen durch „katastrophale” Umwelteinflüsse
}

\author{
E. Ziegelmeier \\ Biologische Anstalt Helgoland (Litoralstation); List/Sylt, Deutscbland (BRD)
}

\begin{abstract}
On mass occurrences of various macrobenthic invertebrates during the phase of recolonization following damages by "catastrophic" environmental influences. In a subsection of the German Bight, qualitative and quantitative studies on population dynamics, conducted during 1961 to 1965 , revealed significant effects of the severe winter of 1962/63 on the abundance of bottom-living species. Catastrophic reductions in abundance, due to very cold winters, are followed by unusually large individual numbers of certain animals during the phase of recolonization. The unusually high abundance of the sedentary polychaete Spbiophanes bombyx recorded after the cold winter of $1962 / 63$ is related to an almost complete absence of Angulus fabula. This mussel represents the type of the so-called "pipetting" animals, and consumes, under normal environmental conditions, the majority of planktotrophic larvae, which are about to settle on the sea bottom.
\end{abstract}

\section{EINLEITUNG}

Unter den abiotischen Umweltfaktoren, die das Vorkommen von marinen Bodentieren im Litoralbereich begrenzen und Fluktuationen mitverursachen, steht direkt oder indirekt die Wassertemperatur an erster Stelle. So können durch langanhaltende, suboptimale Temperaturen, wie in strengen, abnormalen Wintern, bei den epi- und endobenthalen Evertebraten starke Schädigungen eintreten oder verschiedene stenotherme Spezies völlig vernichtet werden. Derartige „Katastrophen"-Winter mit z. T. erheblich unter dem Durchschnitt liegenden Temperaturwerten ließen sich während der letzten 50 Jahre mehrfach beobachten: 1928/29, 1939/40, 1946/47 und 1962/63. Für die beiden Winter vor bzw. zu Beginn des 2. Weltkrieges liegen keine Informationen über die arten- und mengenmäßige Verteilung der Bodenfauna in unserem Meeresgebiet vor.

Als im Herbst 1949 mit den Benthos-Untersuchungen im Ostteil der Deutschen Bucht begonnen worden war, konnte zuerst nur vermutet werden, daß durch den sehr kalten Winter 1946/47 eine Dezimierung des Makrobenthos eingetreten war (ZIEGELMEIER 1953), da einige Bodentierarten anormale Massenentwicklungen zeigten und damit die Phase der Wiederbesiedlung andeuteten. So konnten auf schlickigen Plätzen nordwestlich von Helgoland im. Herbst 1950 sehr dichte Bestände von Aloidis gibba (bis ca. 800 Muscheln auf $0,1 \mathrm{~m}^{2}$ von $6-8 \mathrm{~mm}$ Länge) festgestellt werden, die sich be- 
reits im darauffolgenden Jahr normalisiert hatten (ZIEGELMEIER 1963). Erwähnt sei hierbei, daß dieses Aloidis-Massenvorkommen zu der merkwürdigen „Kordelring ${ }^{\text {- }}$ Bildung bei Schollen (Pleuronectes platessa) geführt hatte (ZregelmeIER 1951).

Bemerkenswert ist, daß etwa zur gleichen Zeit auch bei decapoden Cephalopoda im Nekton auffallende Massenvorkommen beobachtet werden konnten. Nicht selten wurden in einem Hol nach 1/2stiindiger Schleppzeit mit dem Kuttertrawl bei Helgoland im Herbst 1949 z. T. sehr große Exemplare von Loligo forbesi (maximal 70 Tiere) an Deck gebracht. In den Wintermonaten 1950 lagen außergewöhnlich viele Schulpe von Sepia officinalis im Spülsaum. Nach den damaligen, bisher noch unveröffentlichten Beobachtungen, Umfragen, Messungen und eingehenden Untersuchungen des Zustandes der angetriebenen Stücke ergab sich folgendes Bild: Die Fundstellen an den deutschen Meeresstränden waren in ihrer Ergiebigkeit sehr unterschiedlich, am zahlreichsten lagen Schulpe am Strand von Sylt. Die am häufigsten gefundenen SepiaSchalen hatten eine Länge von $60-90 \mathrm{~mm}$, die kleinste maß 35, die größte $210 \mathrm{~mm}$. Abgesehen von den größeren Schulpen über $150 \mathrm{~mm}$, die meist durch Schnabelhiebe von Möwen u. a. nach dem Anspülen beschädigt waren, zeigten die überwiegende Anzahl der Schalen ein frisches Aussehen, z. T. hingen noch Weichkörperfetzen der sicher nicht lange davor abgestorbenen Tiere daran. Dies läßt vermuten, daß mit sehr großer Wahrscheinlichkeit diese Schulpe von Sepien stammen, die im Sommer 1949 von Süden in die Nordsee einwanderten, dort laichten und auf Grund der günstigen Lebensbedingungen das Junggut nicht mehr die Rückwanderung antrat und schließlich der größte Teil im Winter $1949 / 50$ verendete.

Eine Zusammenfassung von Ergebnissen der Bodenfaunauntersuchungen für die Zeit von 1950-1960 (ZIEgelmeIER 1963) ergab einen Hinweis auf die schädigende Wirkung niedriger Temperaturen auf marine Bodentiere durch den kalten Winter 1955/56, der aber in Dauer und Strenge nicht mit dem Eiswinter 1946/47 verglichen werden kann. Im Frühjahr 1956 waren z. B. die Bestände von Echinocardium cordatum, von denen im Herbst 1955 auf den küstennäheren Plätzen bis 170 Herzigel (5-10 $\mathrm{mm}$ Länge) auf $0,1 \mathrm{~m}^{2} \mathrm{im}$ Bodengreifer gefunden wurden, völlig vernichtet.

Eindeutige und stichhaltige Unterlagen für die Bestätigung der Vermutung über die schädigende Wirkung von niederen Temperaturen ergaben die Untersuchungen vor und nach dem strengen Winter 1962/63, der in seiner Länge und Härte mit dem kalten Winter 1946/47 verglichen werden kann. Auch hiernach ist wieder beachtenswert, daß etwa 2 bis 3 Jahre nach den für die Bodentierwelt verheerenden Einwirkungen der strengen Wintermonate Massenvorkommen einer diesmal epibenthalen Wirbellosen-Art festgestellt wurde. Im August/September 1965 und Herbst 1966 sind an den Ostfriesischen Inseln, besonders Norderney, etwas weniger Juist, Baltrum und Langeoog die bisher nicht beobachtete Wuchsform der Bryocoe Electra (Membranipora) pilosa angetrieben worden. In nicht selten bis $1 \frac{1 / 2}{2}$ Meter hohen Spülsäumen lagen dichtgedrängt die etwa tennisballgroßen Moostier-Kolonien (Müller 1966, PrIGge 1967). An den Stränden der Nordfriesischen Inseln, auch auf Sylt, konnten nur vereinzelt die Electra-Bällchen gefunden werden. Die Frage nach der Entstehungsursache dieser Massenvermehrung der Bryozoen wird offen gelassen, nur bei MüLlER finden wir in der Aufzählung der von ihm vermuteten, eventuell die Massenvorkommen begünstigenden Faktoren am Schluß „schlechte Verhältnisse für die Feinde“. Für die An- 
nahme, daß bei der Wiederbesiedlung eines Lebensraumes nach \pm starker Schädigung oder Totalausfall seiner Bewohner die Vernichtungsrate von bestimmten Entwicklungsstadien und die Übetlebenschance nach der Metamorphose im Konkurrenzkampf bei der Eroberung biologischer Nischen eine Rolle spielen und zu Massenentwicklungen einzelner Benthosformen führen können, sprechen die Beobachtungen und Untersuchungsbefunde vor, während und nach dem strengen Winter 1962/63, worauf im vorliegenden Beitrag näher eingangen werden soll.

\section{METHODEN}

Für die Erarbeitung von Einsichten in die Populationsdynamik der Bodenfauna sagen die gelegentlichen, stichprobenartigen Untersuchungen an verschiedenen Stellen in einem Gebiet nichts aus. Sie sind mit photographischen Momentaufnahmen vergleichbar, die nur Aussagen über räumliche, taxonomische Gegebenheiten im Augenblick der Probenentnahme gestatten. Erst die kontinuierlichen Untersuchungen in einem Gebiet über eine längere Zeitspanne bieten die Möglichkeit nach der Auswertung, ähnlich der Teilbildanalyse bei einem Zeitrafferfilm, das dynamische Geschehen in einem. Okosystem erkennbar werden zu lassen.

So werden bei der Biologischen Anstalt Helgoland seit dem Herbst 1949 kontinuierlich bis dato während der 2 Bonitierungsfahrten mit F. K. "Uthörn“ in jedem Jahr, im Frühahr (Ende April/Anfang Mai) und im Herbst (Ende August/Anfang September) routinemäßig auf 19 festgelegten, meist betonnten Stationen im Ostteil der Deutschen Bucht je 2 Proben mit dem van Veen-Bodengreifer 0,1 $\mathrm{m}^{2}$ entnommen.

Gelegentlich durchgeführte Probenserien ergaben bei gleichbleibender Bodenbeschaffenheit verhältnismäßig geringe Streuungen der Arten- und Individuenzahlen pro Flächeneinheit; sie berechtigten zu der Annahme, daß 2 Proben je Station repräsentative Werte erwarten lassen.

Entgegen der früheren Methode, die lebenden Tiere aus dem Fang unmittelbar nach der Probeentnahme an Bord herauszusuchen und sofort zu bestimmen, wurde der gesamte Inhalt der Siebe (kleinste Maschenweite der zwei- bis dreiteiligen Siebsätze $=1 \mathrm{~mm}$ ) in $4-5 \%$ igem Formol-Seewasser fixiert.

Als Lebensbestand (Biomasse) werden das Rohgewicht und das Volumen (mit Hilfe der Wasserverdrängungsmethode) der Formalin-fixierten Tiere ermittelt. Sämtliche Bestandswerte sind nicht auf $1 \mathrm{~m}^{2}$ umgerechnet, sondern beziehen sich auf $0,1 \mathrm{~m}^{2}$.

\section{ERGEBNISSE}

Anlaß zu spezielleren Untersuchungen über die Populationsdynamik nach Schädigungen durch extreme Umwelteinfliusse gaben die Beobachtungen über das Massenvorkommen des Quappwurmes Echiurus echiurus im zähen hellgrauen bis dunklen Schlick, dem teilweise Schill beigemischt war, im Frühjahr und Herbst 1963 auf einigen Stationen vor der Elbe-Mündung. Im Frühjahr 1963 (30. 5.) konnten in Bodengreiferproben $\left(0,1 \mathrm{~m}^{2}\right)$ bei $22 \mathrm{~m}$ Tiefe bis 1005 Echiurus (Durchschnittsgröße ohne löf- 
felförmigen Rüssel 5 $-6 \mathrm{~mm}$ ), an der gleichen Station im Spätherbst 1963 (28. 11.) noch 158 Exemplare mit einer durchschnittlichen Größe (ohne Rüssel) von 20-22 mm

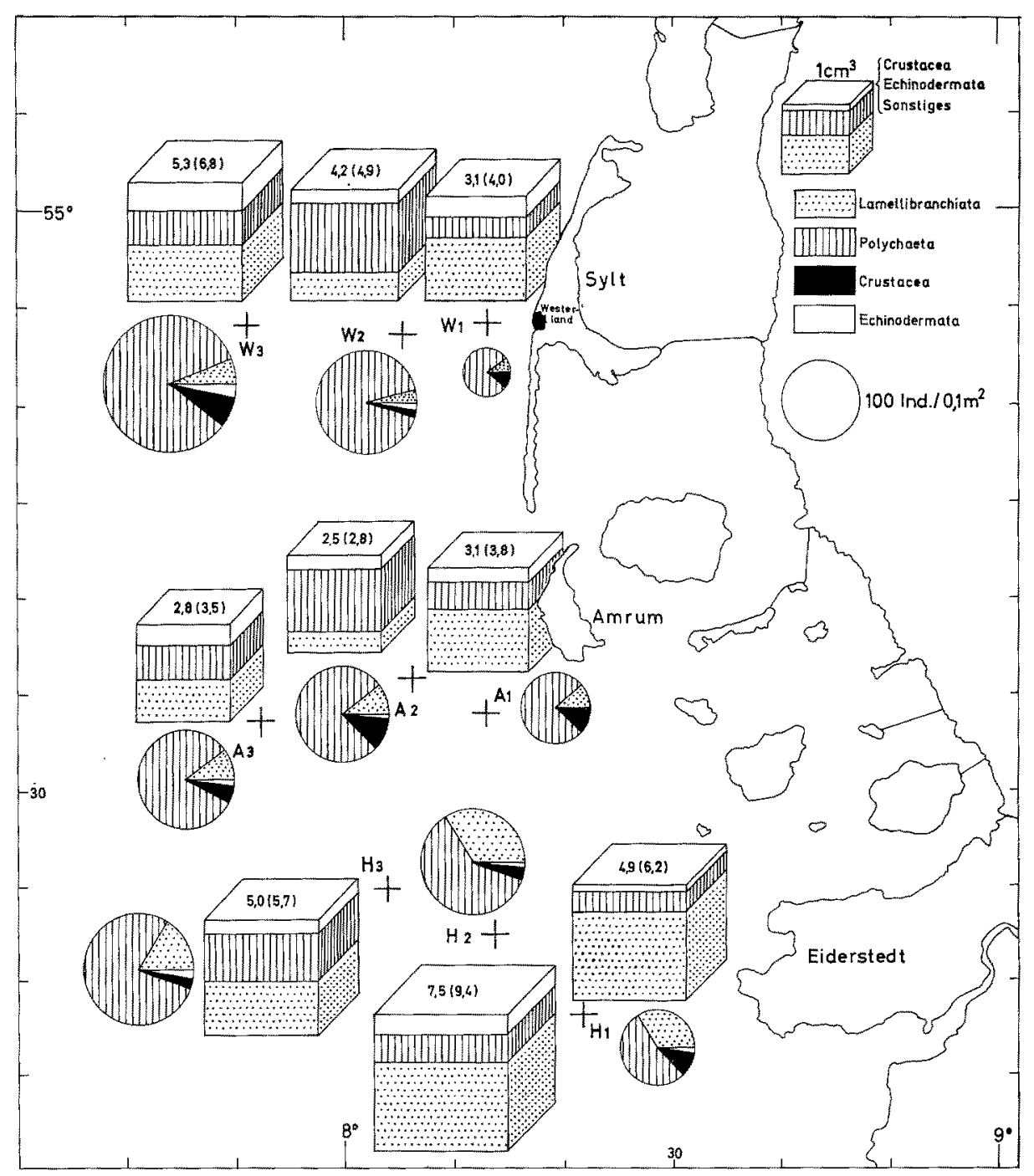

Abb. 1: Untersuchungsgebiet mit den 9 Stationen: Westerland-Schnitt $=W 1$ (reiner gröberer Sand, 10-12 m Tiefe), W 2 (reiner bis schlickiger Sand, 13-15 m), W 3 (reiner bis schlickiger Sand, 19-20 m); Amrum-Schnitt = A 1 (reiner feiner Sand, 9-10 m), A 2 (reiner feiner Sand, 13-15 m), A 3 (reiner bis schlickiger Sand, 19-20 m); Hever-Schnitt $=\mathrm{H} 1$ (reiner bis schlickiger Sand, 9-11 m), H 2 (reiner bis schlickiger Sand, 12-14 m), H 3 (schlickiger Sand, 18-19 m). Durchschnittliche Bestandszahlen (Kreise) und Lebensbestände (Würfel) der vier wichtigsten Klassen der wirbellosen Bodentiere aus allen. Proben während der Zeit von Frühjahr 1961 bis Herbst 1965 auf $0,1 \mathrm{~m}^{2}$ umgerechnet. Die Kreise entsprechen in ihrer Größe den durchschnittlichen Gesamt-Beständen. Die Zahlen in der oberen Würfelfläche = Volumen in $\mathrm{cm}^{3}$, in Klammern dahinter $=$ Gewicht in $\mathrm{g}$ 
festgestellt werden. Bemerkenswert sind Massensiedlungen von Polydora ciliata bis 7850 Tiere $/ 0,1 \mathrm{~m}^{2}$ im Herbst 1963 in dem gleichen Gebiet.

Bei dem Zustandekommen derartig dichter Siedlungen könnte nach den Untersuchungsbefunden die Verringerung der Muschelbestände durch die langanhaltenden niedrigen Temperaturen während des vorangegangenen Winters eine Rolle spielen. Unter normalen Umweltbedingungen wird die Hauptmasse der bodenreifen Larvenstadien besonders von pipettierenden Muscheln aufgezehrt. Durch den Ausfall der Larven-Feinde kommt der größte Teil der jungen Brut auf, und durch die verminderte Nahrungskonkurrenz entstehen günstige Wachstumsbedingungen für einzelne Formen, die dann die erwähnten Massenvorkommen zeigen.

Wieweit diese Annahme durch ähnliche Beobachtungen bei anderen Bodenbeschaffenheiten und anderen Benthosarten mit pelagischen Entwicklungsstadien bestätig werden kann, ließ sich mit Hilfe von quantitativen und qualitativen Bodengreiferproben ermöglichen, die von 9 Stationen aus dem sandigen Mittelteil des Untersuchungsgebietes im Ostteil der Deutschen Bucht vor Sylt, Amrum und Eiderstedt stammen (Abb, 1). Dieses Stationsnetz deckt sich mit dem Hauptverbreitungsareal von Angulus fabula, einer Muschelart, die, mit lang ausstreckbaren Siphonen ausgestattet, imstande ist, die Bodenoberfläche sehr gründlich abzupipettieren. Die ausgewerteten Daten dieser Fänge sind besonders geeignet, (a) die Auswirkungen des sehr strengen Winters 1962/63 auf die epi- und endobenthalen Wirbellosen, (b) Beginn und Verlauf der Wiederbesiedlung und (c) das besonders nach "katastrophalen“ Umweltbedingungen, wie z. B. außergewöhnlich kalte Winter, häufig zu beobachtende Phänomen des Massenvorkommens verschiedener mariner Bodentierformen zu demonstrieren und zu interpretieren.

Mit Ausnahme der küstennahen Grobsand-Station "W " des Schnittes in Höhe von Westerland auf Sylt handelt es sich bei den Fangplätzen des Amrum- und Hever-Schnittes um rein- bis schlicksandige Böden. In diesem Gebiet konnten in der Zeit von 1961 bis 1965 folgende Arten des Makrobenthos festgestellt werden:

Hydrozoa: Tubularia larynx Ellis und Solander, Tubularia indivisa L. Der Aufwuchs mit Hydroiden ist auf den meist stark überspülten und ständig sich umlagernden Sandplätzen spärlich.

An thozoa: Edwardsia danica Calgren.

Nemertin i: mehrere unbestimmte Arten.

Phoron id ea: Phoronis mülleri de Selys-Longchamps.

E chiurida: Echiurus echiurus Pallas.

Poly ch a e ta Er ra ti ia: Gattyana cirrosa Pallas, Harmotboe lunulata Delle Chiaje, Sthenelais limicola Efrers, Pholoe minuta Fabricius, Praegeria remota Southern, Pbyllodoce groenlandica Oersted, Pbyllodoce spec., Eumida (Eulalia) sanguinea OERSTED, Eulalia viridis MürLER, Eteone longa Fabricrus, Nereis longissima Johnston, Nereis spec., Nephtbys caeca Fabricius, Nepbthys bombergi Audourn \& M. Edwards, Nephtbys longosetosa Oerstedt, Nepbthys spec., Ephesia gracilis RATHKe, Glycera capitata OERSTED, Goniada maculata Oersted, Goniada bobrezkii Annenkova, Eone nordmanni Malmgren.

Polychaeta Sedentaria: Aricia cuvieri Audoum \& M.-Edwards, Scolo- 
plos armiger O. F. Müller, Nerine (Scolecolepis) bonnieri Mesnil, Aonides paucibranchiata Southern, Spiophanes bombyx Claparede, Spio filicornis O. F. Müller, Polydora ciliata Johnston, Polydora pulchra Carazzi, Magelona papillicomis F. Müller, Poecilochaetus serpens Allen, Chaetozone setosa MALMGREN, Scalibregma inflatum RATHKE, Ophelia limacina RATHKe, Thoracophelia flabellifera ZiegecmeIER (1955), Ammotrypane anlogaster RAthKe, Travisia forbesii Johnston, Capitella capitata FABricius, Capitomastus minimus LANGERhans, Owenia fusiformis Delle ChIAJe, Myriochele beeri Malmgren, Pectinaria koreni Malmgren, Pectinaria auricoma Müller, Lanice conchilega Pallas.

$\mathrm{Cum}$ a cea: Bodotria scorpioides MonTagu, Psendocuma longicornis Sp. Bate, Lamprops fasciata G. O. SARs, Diastylis ratbkei KröYer, Diastylis bradyi NORMAN.

Mys id a c e: Gastrosaccus spinifer Goes, Paramysis kervillei G. O. Sars.

A mphipoda: Nototropis swammerdami Mrne Edwards, Nototropis falcatus

Metzger, Orchomenella spec., Metopa spec., Ampelisca brevicornis A. Costa, Urothoe poseidonis REIBISCH, Bathyporeia guilliamsoniana BATE, Batbyporeia spec, Perioculodes longimanus BATE \& Westw., Pontocrates altamarinus Bate \& Westw., Pontocrates arenarius Bate, Lembos longipes Lilljeborg, Microprotopus maculatus Norman, Corophium bonelli G. O. Sars, Pariambus typicus KRÖYER.

De c a pod a : Crangon crangon L., Portunus bolsatus Fabricius.

Gastropoda Prosobranchia: Hydrobia ulvae Pennant, Lunatia nitida Donovan, Crepidula fornicata L., Lora turricula MonTAGU.

Gastropoda Opist hobranchia: Cylichna cylindracea Pennant, Pbiline spec.

Lamellibranchiata: Nucula nitida Sowerby, Mytilus edulis L., Astarte triangularis Montagu, Montacuta ferruginosa Montagu, Montacuta bidentata Montagu, Cyprina islandica Montagu, Venerupis pullastra Montagu, Venus gallina L., Petricola pholadiformis LAMarck, Mactra corallina cinerea MoNTAGU, Spisula solida L., Spisula subtruncata DA CosTA, Spisula solidalsubtruncata, Macoma baltica L., Angulus fabula Meuschen, Angulus tenuis DA CostA, Abra alba S. Wood, Donax vittatus DA Costa, Phaxas pellucidus Pennant, Mya truncata L., Aloidis gibba OLrvi, Thracia papyracea Polr.

Echinodermata: Asterias rubens L., Amphipholis squamata Delle Chiaje, Ophiura texturata LAMARck, Opbiura albida Forbes, Echinocardium cordatum Pennant.

Chord a ta: Branchiostoma lanceolatum Patias.

Die nach Abundanz und Lebensbestand (Biomasse) wichtigsten Benthosformen sind Bivalvia und Polychaeta (Abb. 1). Häufigste Muscheln sind die beiden TellinidenArten Angulus fabula und Macoma baltica mit 55 bzw. $20 \%$ aller während der Untersuchungszeit auf sämtlichen Stationen gefundenen Bivalven-Spezies. So gehört das untersuchte Gebiet, wie aus der Abbildung 2 zu erkennen ist, im flachereren östlichen Teil zur eigentlichen Küstengemeinschaft mit Macoma baltica als Leitform und geht mit zunehmender Wassertiefe in die sogenannte Sandgürtel- oder Venus-gallina-Ge- 
meinschatt über. Die Charakter-Form ist nach Abundanz und Frequenz in dieser „Biozönose" aber nicht Venus gallina, sondern die für die feinen, rein- bis schlicksandigen Böden in diesem Tiefenbereich typische Gerippte Tellmuschel Angulus fabula, nach der die Gemeinschaft in unseren Meeresgebieten eigentlich benannt werden sollte. Mit geringerer Abundanz vorkommende Spezies sind Abra alba (10\%), häufiger in den schlicksandigen Böden der Stationen des Hever-Schnittes, weiterhin Montacuta bidentata $(6 \%)$. Die restlichen $9 \%$ verteilen sich auf 17 Arten, die einzeln unter der $2 \%$ Grenze bleiben.

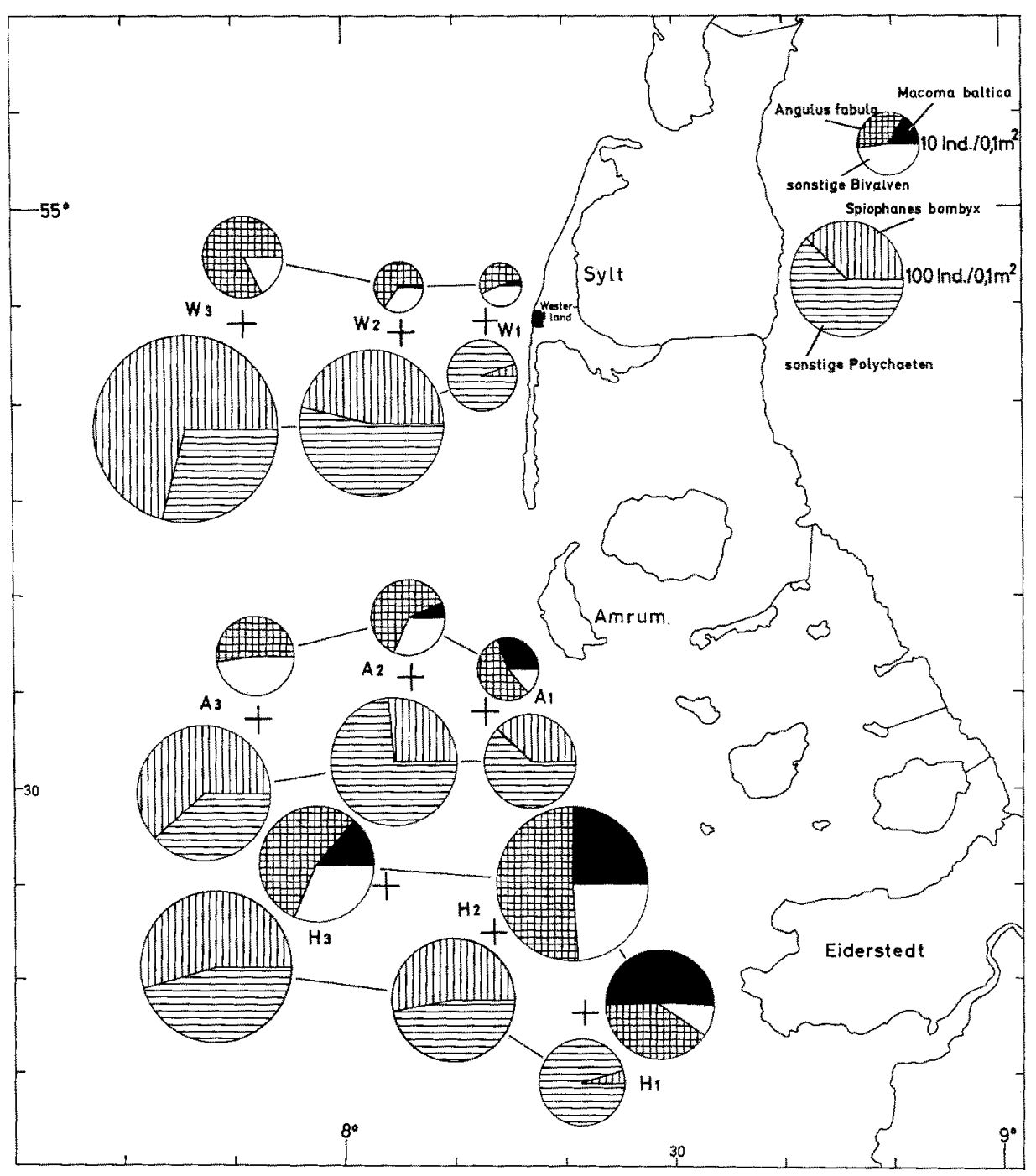

Abb. 2: Durchschnittliche Bestandszahlen von Macoma baltica, Angulus fabula und Spiopbanes bombyx im Verhältnis zu den sonstigen Muscheln bzw. Polychäten für die Zeit von 1961-1965 auf $0,1 \mathrm{~m}^{2}$ umgerechnet 
Die wichtigsten Polychäten in der Reihenfolge der entsprechenden Bestandszahlenanteile sind Spiophanes bombyx $(50,5 \%)$, Scoloplos armiger $(13,3 \%)$, Magelona papillicornis $(11,5 \%)$, Pectinaria koreni $(4,5 \%)$, Lanice concbilega $(3,3 \%)$ und Nephthys hombergi $(2,0 \%)$. Spiophanes bombyx lebt in selbstgebauten dünnen Röh-

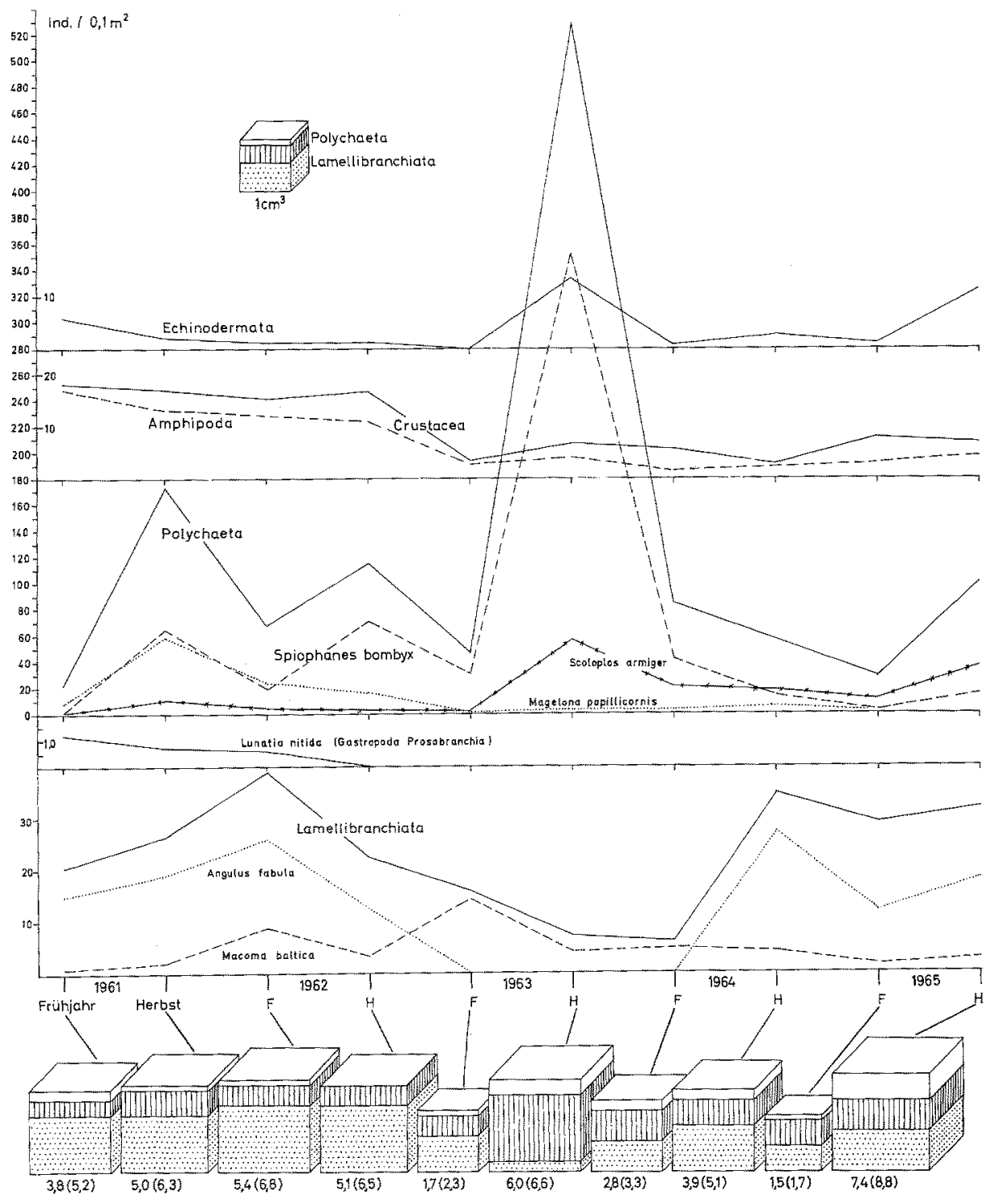

Abb. 3: Durchschnittliche Bestandszahlen und Lebensbestände (untere Würfelreihe) der wichtigsten Bodentierklassen in dem in Abbildung 1 dargestellten Gebiet für die Zeit von Frühjahr 1961 bis Herbst 1965. Die Zahlen unter den Würfeln stellen das Volumen in $\mathrm{cm}^{3} \mathrm{dar}$, in Klammern das Gewicht in $\mathrm{g}$ (sämtliche Werte auf $0,1 \mathrm{~m}^{2}$ umgerechnet) 
ren und bevorzugt wegen des immer benutzten gleichförmigen Baumaterials deshalb \pm reinen Sandboden. Wie Abbildung 2 zeigt, verringern sich die Bestandsdichten nach Süden in den schlicksandigen Stationen. Die Frequenz deckt sich im reinen Sand etwa mit der von Angulus fabula, und die Abundanz nimmt ebenso mit der Tiefe zu.

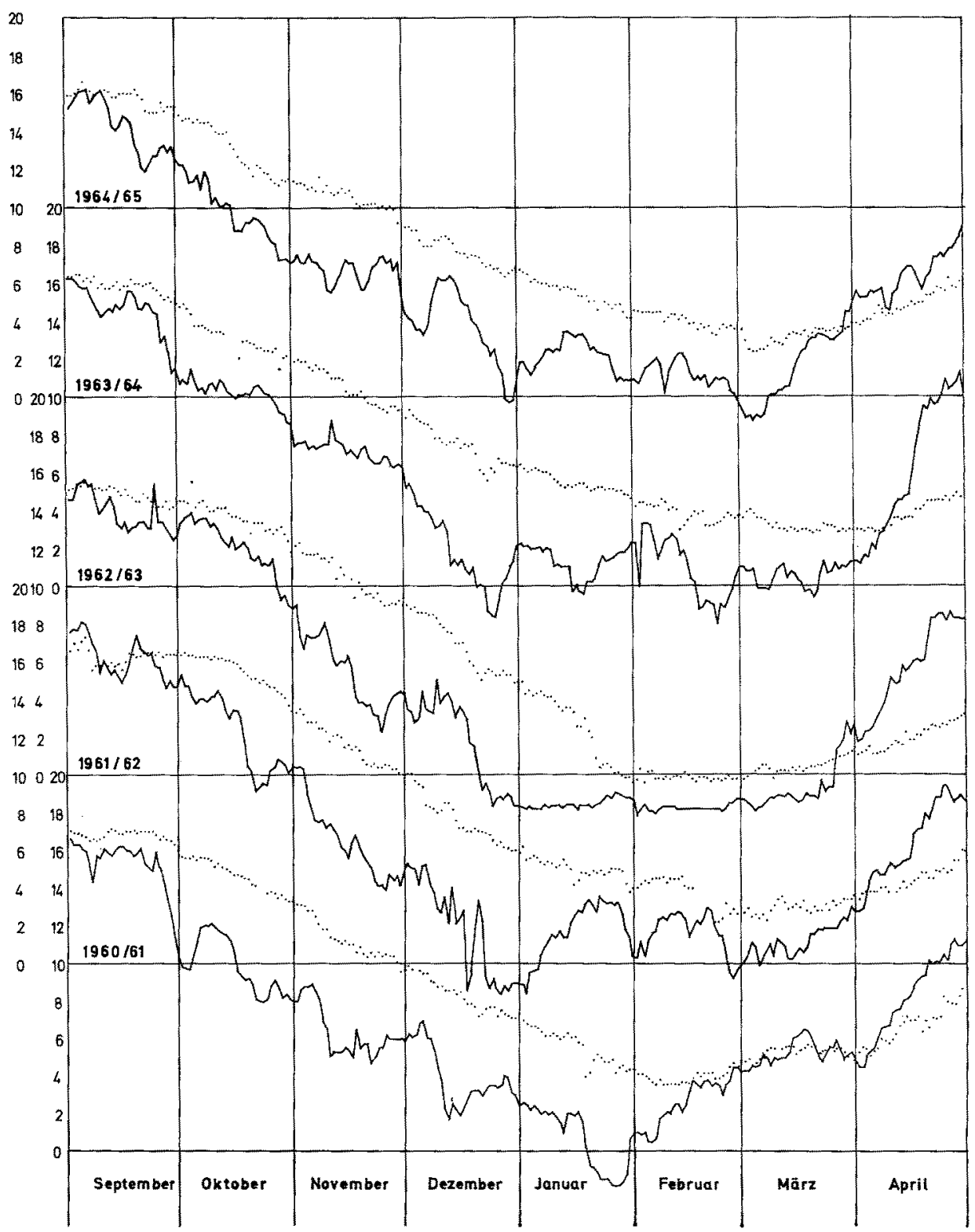

Abb. 4: Oberflächentemperaturen bei List/Sylt (ausgezogene Linie) und am Feuerschiff „P 8“ (punktierte Linie). Mittelwerte von zwei täglich durchgeführten Messungen 
Bei den Crustacea spielen bestandszahlenmäßig die Amphipoda mit 73,6\% die wichtigste Rolle; die häufigsten Arten sind Urotboe poseidonis, Batbyporeia guilliamsoniana und Nototropis swammerdami. Von den restlichen Krebsarten zeigen bei den Cumaceen Ipbinoe trispinosa und Diastylis bradyi, bei Mysideen ein typischer Vertreter des Grobsandes Gastrosaccus spinifer die höchsten Abundanzwerte.

Den Hatuptanteil am Bestand der Echinodermen stellt mit 48,3\% der Herzigel Echinocardium cordatum, der etwas tieferes Wasser bevorzugt und in den sandigen Böden der Außenstationen häufiger anzutreffen ist. Von den Schlangensternen überwiegt die Küstenform Ophiura texturata, nur gelegentlich konnten wenige Exemplare von $\mathrm{O}$. albida in den Bodengreiferproben gefunden werden. Wegen der spärlich anzutreffenden epibenthalen Fauna im Gebiet ist Asterias rubens nur selten in den Fängen zu beobachten.

Die Schädigungen der makrobenthalen Wirbellosen durch den Winter 1962/63 werden mit Hilfe der einfachen graphischen Darstellung (Abb. 3) demonstriert. In Abbildung 4 sind die bei List/Sylt und am Feuerschiff P 8 täglich gemessenen Oberflächentemperaturen jeweils für die Monate September bis einschl. April vom 1. 9. 1960 bis 30. 4. 1965 zusammengestellt. Von den Muscheln am stärksten dezimier wurde die boreal-mediterrane Angulus fabula, am wenigsten die ausgesprochen boreale Macoma baltica (Ziegelmerer 1964).

Lunatia nitida, die glänzende Nabelschnecke, die im Gebiet mit geringer Abundanz, aber ziemlich konstant in den Proben der etwas tieferen Stationen zu finden ist, wurde völlig vernichtet.

Die Polychäten, mit Ausnahme der tubicolen Lanice conchilega, die total ausfiel, überstanden die niedrigen Temperaturen verhältnismäßig gut und die mit den geringsten Bestandsschwankungen und fast konstanter Abundanz vorkommenden Crustaceen sind nicht wesentlich in ihren Beständen geschwächt worden.

Von den Echinodermata konnten besonders bei Echinocardium cordatum erhebliche Ausfälle festgestellt werden.

So war der Gleichgewichtszustand der Lebensgemeinschaften durch den katastrophalen Eingriff völlig gestört, das fast rhythmische Gleichmaß der vor allem jahreszeitlich und fortpflanzungsbiologisch bedingten Bestandsschwankungen unterbrochen. Es bildeten sich biologische Nischen, die, durch den teilweisen oder totalen Ausfall verschiedener Benthosformen entstanden, in der Wiederbesiedlungsphase für deren Beginn und Verlauf von großer Bedeutung sind. Am Anfang der allmählichen Herstellung normaler Bestandsverhältnisse lassen sich, wie Abbildung 3 demonstriert, Massenvorkommen beobachten, die im untersuchten Gebiet durch den Ausfall einer als "Schlüssel“-Spezies geltenden Muschel verursacht worden sind, Angulus fabula.

Während der 3 Untersuchungsfahrten im Frühjahr und Herbst 1963 sowie im Frühjahr 1964 konnten insgesamt 9 lebende A. fabula bei den Stationen $W_{1}, A_{1}, A_{2}$ und $\mathrm{H}_{2}$ im reinen Sand gefunden werden. Die Schalenlängen betrugen 2 bis $5,5 \mathrm{~mm}$. Die Größenverteilung während der gesamten Untersuchungszeit ist in Abbildung 5 dargestellt. In dieser Angulus-fabula-armen Zeitspanne sind bei den Polychäten und bei den Echinodermen deutliche Bestandszahlen-Maxima zu erkennen, die sich nur mit der erwähnten Deutung (Dezimierung der Larven-Zehrer) für das Zustandekommen der Massensiedlung von Echiurus erklären lassen. Bei den Polychaeta ist an der hohen 
Abundanz vor allem Spiopbanes bombyx beteiligt. Diese Spioniden-Art, die wohl in Flecken dichter Besiedlung vorkommt, und, nach den bisherigen zahlreichen Probenauswertungen starke Fluktuationen erkennen läßt, zeigt im Herbst 1963 jedoch so hohe Bestandszahlen, wie sie während der gesamten Untersuchungszeit seit 1950 in

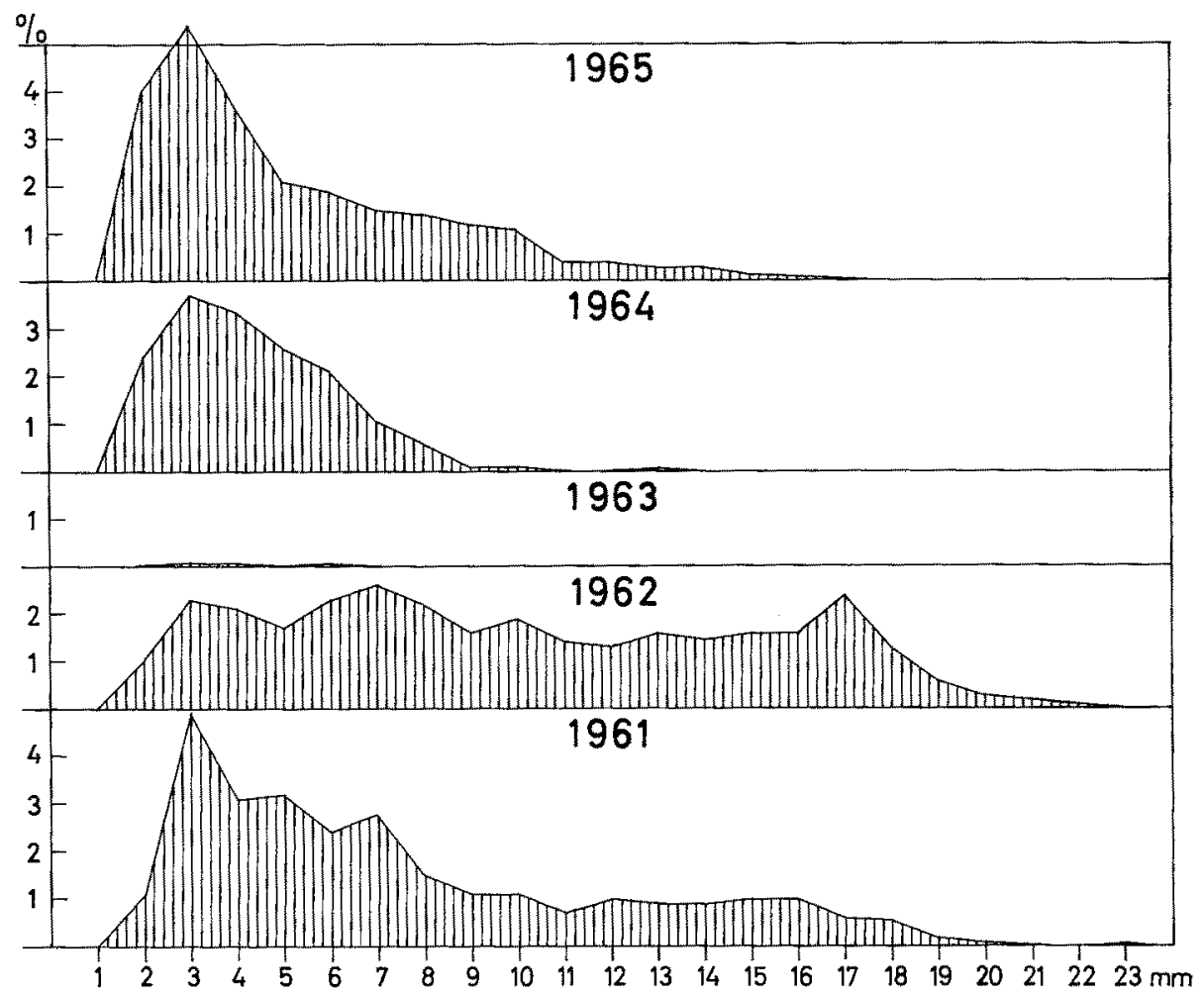

Abb. 5: Größenverteilung von Angulus fabula in den einzelnen Jahren der Untersuchungszeit

diesem Gebiet nicht beobachtet werden konnten (maximal 1614 Individuen in einer 0,1- $\mathrm{m}^{2}$-Bodengreiferprobe). Ein weiteres Argument, das für die Annahme des Larvenschwundes durch Angulus fabula spricht, sind die Abundanzwerte im Herbst 1964. Nachdem im Herbst 1964 die Bestände von $A$. fabula wieder dichter wurden (Abb. 3), ließen die von Spiophanes bombyx stark nach, obwohl nach jahrelangen Beobachtungen (ZIEgelmeier 1963) die Bestandsziffern der Polychaeta allgemein, im besonderen auch von $S$. bombyx, im Herbst ausnahmslos höher liegen als im Frühjahr.

Das Maximum von Scoloplos armiger im Herbst 1963, einer Polychäten-Art mit direkter Entwicklung, könnte mit der Dezimierung verschiedener epibenthaler Tierformen (vielleicht auch Bodenfische), die vermutlich für die wahrscheinlich häufiger erfolgende Zerstörung der auf dem Boden liegenden, mit einem dünnen, ca, $5-7 \mathrm{~cm}$ langen Schleimfaden im Sand verankerten etwa beerengroßen Laichballen mitverantwortlich sind, im Zusammenhang stehen.

Bei dem Bestandzahlen-Maximum der Echinodermen im Herbst 1963 sind haupt- 
sächlich Echinocardium cordatum sowie Ophiura texturata beteiligt, deren Larven durch den fast völligen Ausfall der Zehrer nun bessere Ansatz- und Uberlebenschancen erhielten.

Das biologische Gleichgewicht in diesem Gebiet scheint sich ab Herbst 1965 wieder zu normalisieren, was die Ergebnisse der noch auszuwertenden Proben ab Frühjahr 1966 zeigen sollen.

\section{ZUSAMMENFASSUNG}

1. Massensiedlungen verschiedener mariner Evertebraten nach abnormen, sehr kalten Wintermonaten sind immer wieder beobachtet worden.

2. Anhand von qualitativen und quantitativen Bodenfaunauntersuchungen von 1961 bis 1965 in einem sand- bis schlicksandigen Gebiet im Ostteil der Deutschen Bucht ließen sich die Schädigungen und Totalausfälle der wichtigsten makrobenthalen Wirbellosen infolge des strengen Winters 1962/63 demonstrieren.

3. Es wurde versucht, zur Klärung der Ursachen der außergewöhnlich hohen Bestandszahlen beizutragen. Die Ergebnisse lassen nur die Schlußfolgerung zu, daß durch die fast völlige Dezimierung der im Gebiet am häufigsten vorkommenden, zum Nahrungserwerbstyp der "Pipettierer" zählenden Muschel Angulus fabula, die meisten der bodenreifen planktotrophen Larven einzelner Arten sich nach dem Ansatz mit hoher Abundanz entwickeln können.

Danksaganngen. Der Deutschen Forschungsgemeinschaft danke ich für die Bewilligung von Sachmitteln zur Einstellung einer Hilfskraft bei den Probenauswertungen und Frau B. Dethlefs für die Unterstützung bei den praktischen Arbeiten.

\section{ZITIERTE LITERATUR}

MüLler, C. D., 1966. Seltene Bryozoen-Kugelform in einem Spülsaum. Natur Mus., Frankf. 96, 176-179.

PRIGGE, H., 1967. Uber eine Massenanspülung kugelförmiger Electra-pilosa-Kolonien an den Küsten der südlichen Nordsee in den Jahren 1965 und 1966. Abh. Verh. naturw. Ver. Hamburg 11, 63-78.

Ziegelmeier, E., 1951. Zur Klärung der Cordelringbildung bei Schollen. Fischereiwelt 3 (3), 52-53.

- 1953. Quantitative investigations of the bottom fauna (macrobenthos) in the Helgoland Bight. Annls biol., Copenh. 9 (1952), 140-141.

- 1955. Thoracophelia flabellifera n. sp., ein neuer sedentärer Polychät (Familie Opheliidae) in der Deutschen Bucht. Helgoländer wiss. Meeresunters. 5, 251-257.

- 1963. Das Makrobenthos im Ostteil der Deutschen Bucht nach qualitativen und quantitativen Bodengreiferuntersuchungen in der Zeit von 1949-1960. Veröff. Inst. Meeresforsch. Bremerh. Sonderbd 1 (3. Meeresbiol. Symposion), 101-114.

- 1964. Einwirkungen aes kalten Winters $1962 / 63$ auf das Makrobenthos im Ostteil der Deutschen Bucht. Helgoländer wiss. Meeresunters, 10, 276--282.

Anschrift des Autors: Dr. E. Ziegelmeier Biologische Anstalt Helgoland (Litoralstation)

$2282 \mathrm{List} / \mathrm{Sylt}$

Deutschland (BRD) 